DOI: https://doi.org/10.46296/yc.v6i10edespfeb.0150

\title{
HERRAMIENTAS ESTADÍSTICAS MULTIVARIANTES APLICADAS EN EL ESTUDIO DEL BURNOUT EN DOCENTE: UNA REVISIÓN DE LA LITERATURA
}

\section{MULTIVARIATE STATISTICAL TOOLS APPLIED IN THE STUDY OF TEACHER BURNOUT: A REVIEW OF THE LITERATURE}

\author{
Macías-Romero Francisco Antonio ${ }^{1}$; Useche-Castro Lelly María ${ }^{2}$ \\ ${ }^{1}$ Maestrante del Instituto Posgrado de la Universidad Técnica de Manabí, UTM. Portoviejo, \\ Ecuador. Correo: fmacias1092@utm.edu.ec. \\ ${ }^{2}$ Directora del grupo de investigación GAME. Departamento de Matemáticas y Estadística. \\ Instituto de Ciencias Básicas. Universidad Técnica de Manabí, UTM. Portoviejo, Ecuador. \\ Correo: lelly.useche@utm.edu.ec.
}

\begin{abstract}
Resumen
El Burnout o desgaste profesional, tiende a ser desarrollado en profesionales de diferentes áreas; entre estos, los docentes, centrándose en tres dimensiones: cansancio emocional, despersonalización y baja realización personal. Los métodos estadísticos son fundamentales para el procesamiento de información y la divulgación científica, el estudio se enfoca en exponer las herramientas estadísticas multivariantes empleadas en el análisis del Burnout en docentes; realizando análisis investigativos de relevancia en este ámbito, empleando testimonios de diferentes autores sobre el Burnout en la docencia. Se utilizó una metodología de revisión sistemática de la literatura durante los últimos 10 años para la obtención de un conjunto 10 artículos congruentes con palabras claves como: "Síndrome de Burnout", "Herramientas estadísticas", "Desgaste profesional", "Burnout docente". Entre los resultados de relevancia resaltan herramientas estadísticas multivariantes como: análisis multivariante de la varianza, análisis factorial, análisis de componentes principales, regresión lineal y logística, análisis de correspondencias, ecuaciones estructurales; para identificar y predecir factores generadores del Burnout en la docencia. El análisis teórico de los trabajos elaborados hasta la actualidad, sirve de guía inicial para el uso de las herramientas estadísticas multivariantes más adecuadas para el estudio del Burnout en la docencia como en otras áreas profesionales.
\end{abstract}

Palabras claves: Burnout, métodos multivariantes, docentes, metodología estadística.

\begin{abstract}
Burnout or professional burnout, tends to be developed in professionals from different areas; among these, the teachers, focusing on three dimensions: emotional exhaustion, depersonalization and low personal fulfillment. Statistical methods are essential for information processing and scientific dissemination, The study focuses on exposing the multivariate statistical tools used in the analysis of Burnout in teachers; carrying out relevant research analyzes in this area, using testimonies from different authors about Burnout in teaching. A systematic literature review methodology was used during the last 10 years to obtain a set of 10 congruent articles with keywords such as: "Síndrome de Burnout", "Herramientas estadísticas", "Desgaste profesional", "Burnout docente". Among the relevant results, multivariate statistical tools stand out, such as: multivariate analysis of variance, factor analysis, principal component analysis, linear and logistic regression, correspondence analysis, structural equations; to identify and predict factors that generate Burnout in teaching. The theoretical analysis of the works carried out to date serves as an initial guide for the use of the most appropriate multivariate statistical tools for the study of Burnout in teaching as in other professional areas.
\end{abstract}

Keywords: Burnout, multivariate methods, teachers, statistical methodology.

Información del manuscrito:

Fecha de recepción: 26 de noviembre de 2021.

Fecha de aceptación: 27 de enero de 2022.

Fecha de publicación: 15 de febrero de 2022. 


\section{Introducción}

El Burnout es un término que ha sido objeto de diferentes estudios a nivel mundial, el mismo que se empezó a definir inicialmente hace varios años. Es un término utilizado para referirse a una anomalía caracterizada por agotamiento emocional, despersonalización y baja realización personal, entre otros (Ilaja \& Reyes, 2016). Este síndrome engloba varios factores implícitos en las profesiones que brindan atención y están en contacto con un gran número de personas a la vez.

La importancia de estudiar este síndrome ha ido en aumento durante los últimos años, debido al nivel de exigencias que cada una de las profesiones requiere cada día al adquirir más competencias, que contribuyan a desempeñarse con mayor fluidez en la función ocupacional que cada profesional ejerce, ante esta situación se suele desarrollar ciertos niveles de estrés, mismo que si no son detectados en el momento indicado, posiblemente produzcan la aparición del Burnout (Carlotto \& Palazzo 2006, citado en Vittale et al. 2020). A nivel nacional e internacional se han realizado diversos estudios acerca del síndrome de Burnout en los distintos ámbitos profesionales, en los cuales se han empleado diferentes herramientas estadísticas.

La exploración de la información se la ejecuta por medio de motores de búsqueda 0 base de datos con la inserción de palabras claves que filtran artículos científicos en el área de psicología educativa y herramientas estadística que se emplean en el estudio del síndrome de Burnout en docentes. En cuanto a la calidad de los artículos está se base en función de la revista y su factor de impacto, sea de acceso limitado 0 libre de las investigaciones. La cantidad de publicaciones está comprendida por un total de once. Ante lo expuesto, basándose en la siguiente premisa de investigación ¿cuáles son las herramientas estadísticas multivariantes empleadas en el estudio del síndrome de Burnout en docentes?, se planteó como objetivo, exponer las herramientas estadísticas multivariantes empleadas en el análisis del síndrome de Burnout en docentes. 


\section{Metodología}

El conglomerado de artículos base se obtiene a través de una búsqueda bibliográfica mediante el uso de sitos web como son: Google Scholar, Dialnet, Scielo, Resarch Gate, Science Direct, entre otros.

Para el filtrado de la información se emplean palabras clave como, "Síndrome de Burnout", "Herramientas estadísticas", "Burnout Docente", "Desgaste profesional", con este tipo de configuración permite acceder a trabajos relevantes, donde inicialmente se obtiene acceso a los títulos de 276 artículos, entre los cuales se citan 35 artículos; la investigación se limitó a las técnicas multivariantes, quedando un total de 10 publicaciones, que han sido consideradas pertinentes $y$ relevantes para este trabajo, las que fueron sometidas a un proceso riguroso de revisión, análisis y descripción; los 266 restantes se desecharon porque no se ajustaban al objetivo de estudio; Así mismo se evaluó la calidad de los artículos y la frecuencia de las herramientas estadísticas multivariantes utilizadas dentro de una misma área de estudio del síndrome de Burnout, analizando la importancia y vialidad de dichas herramientas estadísticas en el desarrollo de futuras investigaciones.

\section{Revisión de la literatura}

\section{- Síndrome de Burnout}

Según Saborio \& Hidalgo 2015; citado en Quispe, (2017), la Organización mundial de la salud (OMS) en el año 2000 declaró al "síndrome de Burnout como un factor de riesgo laboral que podría afectar la calidad de vida, saluda mental e incluso perder la vida" (pág. 12). E Burnout ha sido investigado en diferentes áreas, por ejemplo, en los médicos por (Tafoya et al. 2020) en el caso de los policías (Montero et al. 2020), mientras en el ámbito deportivo por (García-Jarillo et al. 2020), por otra parte, en el régimen penitenciario se encuentra el trabajo realizado por (Bruce et al. 2019), y en los funcionarios dedicados a la labor de cajero en entidades financieras (Rodríguez et al. 2018).

El Burnout es caracterizado por ser un síndrome a desarrollarse en las profesiones que están sometidos a grandes presiones laborales, como es el caso de los docentes. Las 
personas que desarrollan este síndrome tienden a presentar sentimientos y actitudes negativas hacia el propio rol profesional y hacia las personas con las que labora, así como por el hecho de estar agotado emocionalmente.

El síndrome de Burnout o también conocido como desgate profesional es un factor latente el cual tiende a ser desarrollado en los profesionales de diferentes áreas. Es un síndrome de índole psicológico que es desarrollado en base al entorno laboral, respondiendo a múltiples estresores crónicos interpersonales. Ante el incremento del nivel de exigencias en las diferentes profesiones y por el fortalecimiento de las competencias que faciliten la fluidez del buen desempeño profesional, se vuelven vulnerables y propensos a desarrollar diferentes niveles de estrés, lo cual se torna un factor latente ante la posible aparición del Burnout (De Souza et al. 2021). Entre los factores externos pueden destacarse los problemas económicos o familiares, miedo, exceso de trabajo, entre otros. Mientras que de los factores internos se resaltan los sentimientos de inferioridad, las enfermedades, problemas psicológicos, dolor corporal, etc(Orozco et al. 2021).

El cansancio es un factor común en las profesiones y entre ellos los educadores donde pueden producirse alteraciones emocionales generalmente involucradas por la sociabilidad y la disciplina, además de la labor docente y del proceso de interaprendizaje en las diferentes fases de la escolarización (Gutiérrez \& Naranjo, 2020).

\section{- Herramientas estadísticas}

La estadística es una ciencia que brinda un sinnúmero de bondades por la amplia variedad de herramientas que pone a disposición para ser utilizadas en los diferentes campos del saber, con el propósito fundamental de aportar en la solución a diversas problemáticas con cierto nivel de confianza (Saucedo-Moreno \& FenigRodríguez, 2019). Entre las diferentes herramientas estadísticas existentes, varias se usan en la descripción de individuos y fenómenos, otras para validar la consistencia interna de instrumentos previamente elaborados, algunas para inferir y poder realizar predicciones, construcción de 
modelos, entre otras, basándose cada una en criterios o parámetros propios de cada herramienta (Castro, 2019).

Dentro del ámbito psicológico es frecuente el uso de las herramientas estadística univariantes, bivariantes y multivariantes (Carvajal \& Gómez, 2014), con la finalidad de facilitar el procesamiento de la información en estudios de diversos síndromes psicológicos inherentes a ser desarrollado en el ser humano, en la cual están inmersas un gran conjunto de variables (QuilliganaGuachi et al. 2021).

Aquí se destaca las herramientas estadísticas multivariantes utilizadas en el estudio del síndrome de Burnout en docentes; teniendo presente que la estadística multivariante tiene sus inicios en 1809 con Gauss por medio de la regresión lineal y luego en 1900 con otros estadísticos como Markov, estos métodos fueron integrando un sinnúmeros de técnicas para el análisis de datos, los mismos que brindan una gama de bondades que facilitan la reducción de un gran conjunto de variables a un número relativamente pequeño con pocos componentes que las simplifican, reconociendo las posibles correlaciones existentes entre las variables (Closas et al. 2013).

Entre las herramientas estadísticas multivariante utilizadas en indagación del Burnout en la profesión docente, se tienen las empleadas en los trabajos de:

Menghi \& Rodríguez (2019) buscaron las diferencias existentes entre los valores en cada una de las dimensiones que tiene el síndrome de Burnout en un grupo de educadores los cuales presentaban - no algún problema de salud, apoyándose en estadística descriptiva, y herramientas de estadística multivariada como lo es el análisis factorial exploratorio. Haciendo uso de estas herramientas estadísticas se estableció diferencias significativas en las dimensiones de cansancio emocional y despersonalización en el grupo de docentes, mientras los docentes con algún problema de salud presentan los valores más altos en los niveles de Burnout.

Mesurado \& Laudadío (2019) en la investigación el Burnout y su relación con las variables: experiencia laboral, capital psicológico y 
engagement, emplearon estadístico descriptivos, test de normalidad de Kolomogorov-Smirnov, test de Kruskal-Wallis para las variables no paramétricas, análisis multivariante de la varianza MANOVA, análisis de regresión; con el apoyo de esta herramientas estadísticas se pudo reconocer que los docentes con más años de antigüedad en la docencia presentan mayores niveles de capital psicológico y mayores niveles de absorción por el trabajo, no existen diferencias significativas entre la experiencia académica con los niveles de Burnout; mientras que dos dimensiones del capital psicológico y una del engagement impiden el desarrollo del síndorme de Burnout.

Sánchez-Oliva et al. (2014) investigaron el Burnout y la motivación en docentes de educación física, apoyándose de estadística descriptiva, análisis de consistencia interna, análisis de correlaciones binarias, análisis de regresión lineal múltiple; con el uso de estas herramientas estadísticas se logró determinar que la variable frustración por la competencia era predictora positiva de la desmotivación, el cinismo docente y el agotamiento emocional; mientras que negativamente la eficacia profesional con la motivación intrínseca, la frustración autónoma predice positivamente al agotamiento emocional y negativamente a la eficacia profesional, la frustración en las relaciones sociales predijo positivamente a la eficacia profesional.

Granados et al. (2019) se dedicaron a indagar acerca los diferentes factores que permiten predecir la despersonalización y baja realización personal por medio de causas como el estrés y la depresión en el personal docente, utilizando diferentes herramientas estadísticas: el análisis descriptivo, regresión logística usando el estadístico de Wald, para la estimación de los parámetros del modelo se emplearon los odd ratio (OR) y finalmente se realizó la evaluación en cuanto al ajuste y calidad del modelo obtenido a través del indicador R^2 de Nalgerkerke; con lo cual se logró identificar una alta probabilidad de padecimiento de despersonalización, mientras que la baja realización personal aumenta con el aumento del estrés, ansiedad y depresión. 
Mirón et al. (2018) estudiaron el Burnout y el sentido del humor que se explican a través de variables latentes empleando análisis factorial de componentes principales, análisis de consistencia interna, test de esfericidad de Bartlett a través del $\mathrm{KMO}$ y test $\mathrm{X}^{\wedge} 2$; con el apoyo de estas herramientas estadísticas se encontró la existencia de correlación inversa entre las variables Burnout y sentido del humor.

El análisis por componentes principales es una herramienta de la estadística multivariante que permite identificar como se agrupan un sinnúmero variables en una misma dimensión de un gran conjunto de datos, reduciendo el número de variables (De Coster 1998, citado en Mavrou, 2015).

Pedditzi et al. (2020) en la investigación del Burnout y el estrés en docentes, se apoyaron en el modelo estadístico de múltiples indicadores y múltiples causas (MIMIC), haciendo uso de estos modelos se identificó que el cansancio emocional $y$ despersonalización son predichos por los factores: conflicto interpersonal, la interfaz entre trabajo y el hogar.
Fuster-Guillén et al. (2019) se dedicaron a indagar acerca del análisis factorial confirmatorio en el desgaste profesional de los docentes, en el cual aplicaron varias herramientas estadísticas entre las cuales están: análisis de consistencia interna para verificar la fiabilidad en las escalas del Burnout, se realizó test de normalidad en conjunto con la curtosis para comprobar la distribución de los datos, el método de distribución libre asintótica para la estimación de los parámetros del modelo factorial confirmatorio, con el apoyo de estas herramientas se encontró que los docentes de la facultad de ingeniería presentan menor desgaste profesional en relación a los docentes de la facultad de Educación.

Manrrique \& Avendaño (2021) estudiaron el Burnout docente y la relación que este presenta con el acoso laboral, apoyándose en distribución de frecuencias para la parte sociodemográfica, test de consistencia interna con el uso del Alfa de Cronbach, para verificar la distribución de los datos se aplicó el test de normalidad de KolmogorvSmirov en conjunto el test de Liliefors 
para la corrección de la significancia en la distribución, pruebas de $X^{\wedge} 2$ para determinar la relación entre variables, modelo de ecuaciones estructurales, índice de validación cruzada esperada (ECVI), Chicuadrado para los grados de libertad (CMIN), error cuadrático medio de aproximación (RMSEA) y los coeficientes para comparaciones (CFI). Utilizando en esa diversidad de herramientas estadísticas se logró identificar que el Burnout es explicado en un $64 \%$ por la variable acoso laboral.

Arias et al. (2019) ejecutaron un estudio comparativo y psicométrico acerca del síndrome de Burnout en docentes de escuela y universidad se usó estadísticos descriptivos, el modelo factorial confirmatorio usando para la estimación de los parámetros el método de mínimos cuadrados ponderados y para la evaluación de la calidad y ajuste del modelo se utilizó el índice del error cuadrático medio de aproximación (RMSEA), índices de coeficientes para comparaciones (CFI), la raíz residual estandarizada cuadrática media(SRMR) y el omega para la fiabilidad del modelo; con lo cual se encontró que existen diferencias significativas en función del estado civil, sexo y nivel de enseñanza; resaltando que existe mayor nivel de cansancio emocional en los docentes de escuela y los profesores universitarios, presentando niveles moderados de despersonalización los mismo que mostraron correlaciones negativas con el números de hijos y el Burnout.

Fernández-Puig et al. (2015) se enfocaron a valorar la salud de los docentes en el ambiente laboral en centros educativos privados, apoyándose en estadística descriptiva, análisis de consistencia interna, análisis factorial exploratorio, análisis de la varianza (ANOVA) con el contraste de Bonferroni, estadístico KMO, test de Barlett, para la extracción de los factores se utilizó método de mínimos cuadrados ponderados con rotación Oblimin. Además se empleó el estadístico de Tucker-Burt, a renglón seguido se aplicó análisis factorial confirmatorio por el método de máxima verosimilitud, para el ajuste y la calidad del modelo aplicaron los siguientes índices: índice de coeficientes para comparaciones (CFI), el índice de ajuste de Bollen(IFI), índice de 
bondad de ajuste (GFI), índice ajustado de calidad en el ajuste (AGFI), índice del error cuadrático medio de aproximación (RMSEA) y el índice $X^{\wedge} 2$. Para la comparación de los modelos se utilizó el criterio de información de Akaike (AIC), empleando estas herramientas estadísticas se evidenció que todos los factores de los instrumentos presentan altos índices de fiabilidad.

\section{Conclusiones}

La investigación consistió en la realización de un análisis bibliográfico de una gama de artículos científicos, los cuales se ejecutaron en distintos tiempos y en diferentes instituciones a nivel mundial.

Las técnicas multivariantes, como el análisis factorial, análisis de regresión componentes principales y ecuaciones estructurales, permitieron obtener resultados que con las técnicas univariables y bivariables no se obtenían, tales como el estudio de manera simultánea de los factores que afectan el síndrome de Burnout y cuáles de ellos prevalecían sobre los demás. Por otra parte, se puede evidenciar la variedad de herramientas estadísticas empleadas, facilitando que el investigador pueda mostrar datos minimizando la incertidumbre en la justificación de tesis o conclusiones.

Las herramientas multivariantes tienen una gran aplicación en el estudio del Burnout, teniendo presente que, en este síndrome, el investigador maneja un conjunto amplio de variables. Por ende, es fundamental indicar que el investigador tiene la libertad para poder escoger las herramientas estadísticas acorde al estudio a desarrollar, recalcando que la selección de la metodología se convierte en la parte primordial la cual va a tener incidencia directa en la calidad de los resultados que se obtendrán, por este motivo una consideración de relevancia es tener conocimiento de las ventajas y escenarios en los cuales se realizará la aplicación, para lo cual el investigador deberá tener siempre presente la técnica estadística a utilizar inclusive desde antes de la recolección de la información, desde el mismo planteamiento de la investigación. 


\section{Bibliografía}

Arias, W., Huamani, J. C., \& Ceballos, Kk. (2019). Síndrome de Burnout en profesores de escuela $y$ universidad: un análisis psicométrico y comparativo en la ciudad de Arequipa. Propósitos

Representaciones, 7(3), 72110.

https://doi.org/http://dx.org/10 .20511/pyr2019.v7n3.390

Bracco, L. B., Váldez, R. O., Wakehami, A. N., \& Velázquez, T. (2019). Síndrome de Agotamiento Profesional y Trabajadores Penitenciarios Peruanos. Una Mirada Cualitativa a los Factores Institucionales y Sociales. Revista Colombiana de Psicología, 28(1), 13-28. https://doi.org/10.15446/rcp.v 28n1.66056

Carvajal, C. C., \& Gómez, C. X. Y. (2014). Factor analysis of forced-choice items: A review and an example. Revista Latinoamericana de Psicología, 46(1), 24-34. https://doi.org/10.1016/s0120 -0534(14)70003-2

Castro, E. M. M. (2019). Bioestadística aplicada en investigación clínica: conceptos básicos. Revista Médica Clínica Las Condes, 30(1), 50-65. https://doi.org/10.1016/j.rmclc .2018.12.002

Closas, H. A., Arriola, E. A., Kuc, C. I., Amarilla, M. R., \& Jovanovich, E. C. (2013). Análisis multivariante, conceptos y aplicaciones en Psicología Educativa y Psicometría. Enfoques, XXV (1), 65-92. https://www.redalyc.org/pdf/2 59/25930006005.pdf

De Souza, S. P., Formés-Vives, J., Unda-Rojas, S. G., PereiaJunior, G. A., \& Cardoso, L. (2021). Análisis factorial confirmatorio del Maslach Burnout Inventory - Human Services Survey en profesionales de la salud de los servicios de emergencia. Revista Latino-Americana de Enfermagem, 29, 2-9. https://doi.org/10.1590/15188345.3320 .3386

Fernández-Puig, V., Mayayo, J. L., Chamorro, A. C., \& Tejedor, C. V. (2015). Evaluando la salud laboral de los docentes de centros concertados: El Cuestionario de Salud Docente. Journal of Word and Organizational Psychology, 31(3), 175-185. https://doi.org/10.1016/j.rpto. 2015.07.001

Fuster-Guillén, D., Jara-Jara, N., Ramírez-Asís, E., Maldonado-Leyva, H., Norabuena, R. F., \& García, A. G. (2019). Desgaste 
ocupacional en docentes universitarios mediante el modelo factorial confirmatorio. Propósito y Representaciones, 7(3), 198230.

https://doi.org/10.20511/pyr2 019.v7n3.389

García-Jarillo, M., De Francisco, C., Ortín, F. J., \& Garcés de Los Fayos, E. J. (2020). Sintomatología del síndrome de burnout en deportistas: un estudio con metodología Delphi. Cuadernos de Psicología Del Deporte, 20(3), 15-25.

https://doi.org/10.6018/cpd.36 7531

Granados, L., Aparisi, D., Inglés, C. J., Aparicio-López, M. D. P., Fernández-Sogorb, A., \& García-Fernández, J. M. (2019). ¿Predicen los factores de depresión, ansiedad y estrés la dimensión de la despersonalización y la baja realización personal en el profesorado? European Journal of Child Development, Education and Psychopathology, 7(1), 83. https://doi.org/10.30552/ejpad .v7i1.91

Gutiérrez, G., \& Naranjo, E. (2020). Síndrome de Burnout en el personal docente de la Facultad Ciencias de la Salud de la Universidad Técnica de Ambato. Enfermería Investiga, 5(3), 19-26. https://doi.org/10.31243/ei.uta .v5i3.905.2020

Ilaja, B., \& Reyes, C. (2016). Burnout y estrategias de inteligencia emocional en profesores universitarios: Implicaciones en la salud laboral educativa. Psicología Desde El Caribe, 33(1), 31-46. https://doi.org/10.14482/psdc. 33.1.8081

Manrrique, A. M., \& Avendaño, B. L. (2021). Relación entre Mobbing y Síndrome de Burnout en Docentes. Revista Mexicana de Investigación En Psicología, 13(1), 115-130. https://www.revistamexicanad einvestigacionenpsicologia.co m/index.php/RMIP/article/vie w/348/226

Mavrou, I. (2015). Análisis factorial exploratorio: Cuestiones conceptuales y metodológicas. Revista Nebrija de Lingüística Aplicada a La Enseñanza de Lenguas, 19, 71-80. https://doi.org/10.26378/rnlael 019283

Menghi, M. S., \& Rodríguez, L. M. (2019). Diferencias en los valores de las dimensiones del burnout en educadores con $y$ sin síntomas 0 problemas de salud. Propósito y Representaciones, 7(3), 179188.

https://doi.org/10.20511/pyr2 019.v7n3.338 
Mesurado, B., \& Laudadío, J. (2019). Experiencia profesional, capital psicológico y engagement. Su relación con el burnout en docentes universitarios. Propósitos y Representaciones, 7(3), 1226.

https://doi.org/10.20511/pyr2 019.v7n3.327

Mirón, G. M., García, A. S., \& Ramos, J. H. (2018). Variables latentes que explican el sentido del humor y el síndrome de Burnout: Un estudio empírico piloto en profesores y personal administrativo en una institución de educación media superior. Revista Electrónica de Psicología Iztacala, 21(4), 1662-186. http://revistas.unam.mx/index .php/repi/article/view/68035/6 0007

Montero, K. L., Olarte, G. A., \& Hernández, Á. U. (2020). Síndrome del burnout en polícias de Colombia y su relación con el sistema de beneficios e incentivos. Revista Logos, Ciencia \& Tecnología, 12(2), 32-43. https://doi.org/10.22335/rlct.v $12 \mathrm{i} 2.1161$

Orozco, Z. L., Raymondi, K. R., Orozco, S. M., \& Bohórquez, S. K. (2021). Análisis de los valores interpersonales y su relación con el síndrome de Burnout en docentes de instituciones educativas del cantón Simón Bolívar. Psicología Unemi, 5(9), 118126.

https://doi.org/10.29076/issn.

2602-

8379vol5iss9.2021pp118-

$126 \mathrm{p}$

Pedditzi, M. L., Nicotra, E. F., Nonnis, M., Grassi, P., \& Cortese, C. G. (2020). Teacher Stress and burnout: a study using MIMIC modelling. Electronic Journal of Applied Statistical Analysis, 13(3), 739-757.

https://doi.org/10.1285/i20705 948v13n3p739

Quilligana-Guachi, L. C., EspinozaBeltrán, P. S., \& Siza-Siza, L. A. (2021). El progreso de la Estadística y su utilidad en la Evaluación del desarrollo Académico. Dominio de Las Ciencias, 7(4), 1645-1651. https://doi.org/http://dx.doi.org /10.23857/dc.v7i3.2126

Quispe, Y. (2017). Síndrome de Burnout en docentes de la esculea de administración. Universidad Nacional Del Altiplano/ Revista de Investigaciones de La Escuaela de Posgrado, 6(1), 11-18.

https://doi.org/10.26788/riepg .2017 .23

Rodriguez, S. F., Gómez, A., \& Suasnavas, P. (2018). Cansancio emocional, despersonalización y 
realización en trabajadores del sector bancario. INNOVA Research Journal, 3(1), 1018.

https://doi.org/10.33890/innov a.v3.n1.2018.324

Sánchez-Oliva, D., Sánchez-Miguel, P. A., Pulido, J. J., López, J. M., \& Cuevas, R. C. (2014). Motivación y burnout en profesores de educación física: incidencia de la frustración de las necesidades psicológicas básicas. Cuadernos de Psicología Del Deporte, 14(3), 75-82.

https://doi.org/10.4321/s1578 $-84232014000300009$

Saucedo-Moreno, E. M., \& FenigRodríguez, J. (2019). Estadística en cirugía, como entender y aplicar conceptos básicos. Cirugia y Cirujanos (English Edition), 87(6), 692697.

https://doi.org/10.24875/CIRU .19000487

Tafoya, S. A., Jaimes-Medrano, A. L., Carrasco-Rojas, J. A., Mújica, M. L., RodríguezMachain, A. C., \& Ortiz-León, S. (2020). Asociación del acoso psicológico con el desgaste profesional en médicos residentes de la Ciudad de México. Investigación En Educación Médica, 9(35), 18-27. https://doi.org/10.22201/facm ed.20075057e.2020.35.2020

4

Vittale, F. A., Velázquez, S. A., \& Ortiz, I. G. (2020). Frecuencia del síndrome de Burnout en residentes de medicina interna del hospital de clínicas. Revista Virtual de La Sociedad Paraguaya de Medicina Interna, 7(2), 72-79. https://doi.org/10.18004/rvsp mi/2312-3893/2020.07.02.72 